\title{
HEP interactive activities in high schools in the framework of the CREATIONS project
}

\author{
Christine Kourkoumelis ${ }^{1}$ \\ Physics Faculty, University of Athens \\ Panepistimioupoli, Ilissia 15771, Greece \\ E-mail: hkourkou@phys . uoa.gr
}

\section{Stylianos Vourakis}

Physics Faculty, University of Athens

Panepistimioupoli, Ilissia 15771, Greece

E-mail: s_vourakis@phys.uoa.gr

\section{Sofoklis Sotiriou}

Research and Development Department, Ellinogermaniki Agogi

Dimitriou Panagea Str.15351 Pallini, Greece

E-mail: sotirioudea.gr

CREATIONS is a Horizon 2020 European Union outreach project, which aims to increase young people's interest in science. In the framework of CREATIONS we have been developing advanced digital tools and educational resources in order to facilitate the introduction of HEP in high schools and at the same time we have performed a number of interventions in Greek schools which take the form of half-day masterclasses. The students, working in their schools' computer labs, have the opportunity to use the inquiry based science education scenaria which have embedded interactive tools in order to get familiar with CERN and its research. The students' work is monitored step by step by a specially designed integrated assessment system.

We summarize the available tools as well as the results of the assessment analysis which provide a clear indication that the interventions in the classrooms have been successful and that the students are demonstrating deep involvement and high problem solving skills.

The European Physical Society Conference on High Energy Physics

5-12 July, 2017

Venice

\section{${ }^{1}$ Speaker}

(C) Copyright owned by the author(s) under the terms of the Creative Commons

Attribution-NonCommercial-NoDerivatives 4.0 International License (CC BY-NC-ND 4.0). 


\section{Introduction}

CREATIONS [1] is a three-year long European Union funded project, which aims to develop an engaging science classroom. Sixteen partners from ten European countries develop creative approaches based on science, art and their combinations to ignite the students' interest in science. The project, which is coordinated by the University of Bayreuth, is now in its second year and a variety of events have already taken place (Global science opera, Learning through theater etc.). We have developed a number of digital tools and educational scenaria for all grades of high school in order to facilitate the introduction of HEP in high schools. The students, working in their schools' computer labs, have the opportunity to use the inquiry based science education resources which have embedded tools for analysing data from the ATLAS experiment or to discovering how the LHC works through playful learning. The students' work is monitored step by step by a specially designed integrated assessment system which consists of three different layers which are described in section 3.

In addition to the resources addressed to high school students we have also developed a special version of the HYPATIA on-line event analysis tool to be used as a lab course for third year undergraduate physics students.

\section{Mini masterclasses at schools}

In the framework of CREATIONS we have performed a number of events in Greek schools which take the form of half-day masterclasses trying to fit them in the tight schedule of Greek schools. The so-called mini masterclasses consist of two parts. The students are introduced to a specially designed educational scenario consisting of five different phases. More specifically the first part of the mini-masterclass starts with the description of the first two phases of the inquiry learning scenario: "Orienting and asking questions" and "Hypothesis Generation and Design". At the end of each phase they are asked to answer relevant questions measuring their pre-activity understanding. Then, they are given specific information on tasks

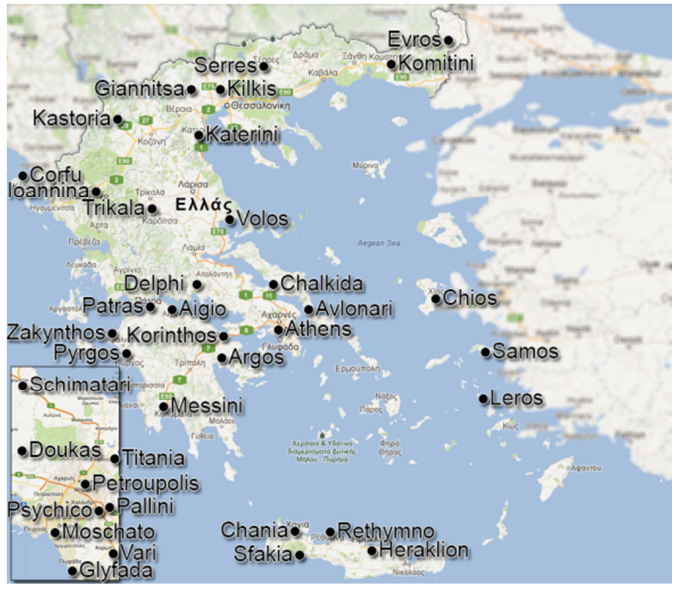

Figure 1. Location of schools where the mini masterclasses took place in Greece that they will have to perform during the hands-on activity which will follow and which is embedded in the "Planning and Investigation" phase. This is the main phase where the students spend most of their time. The resource on which they work is chosen according to their age group.

Following the end of the hands-on activity, the students answer more questions and proceed to the second part. In the second part they discuss their results with their fellow students, teachers and researchers, they present their findings and answer the final two questions of the fourth phase. Finally the intervention often ends with a virtual visit to one of the main experiments of the LHC and a long question and answer session with the researcher connected virtually from CERN, where the students learn "live" about the detection techniques and the experiment operation. 
During recent years the mini masterclasses have been implemented in more than 50 school environments around Greece as shown in Figure 1.

\section{Available resources}

The available resources which we have developed and used in high schools in the last years are the following:

A scenario called "ALL that matters" [2] for kids of age 10-12. This educational activity has embedded in its third phase two of the CERNland games. The first one called "Microboy" teaches kids how to capture protons, neutrons and electrons and make atoms from the simplest ones up to the more complicated (Hydrogen to Carbon). This can be used in the context of a chemistry class as well. The second part of the scenario involves a more complicated game called "TOTEM" which through playful learning teaches the kids about quarks, the structure of the nucleons and the particles keeping the quarks together.

For junior high school students (ages 12-15) the available resource called "Let's accelerate particles" [3] has embedded in its third phase the CERNland's "LHC game". This way the students learn interactively how to accelerate charged particles, bend them by magnetic fields and finally focus them using more complicated magnetic fields.

For senior high school students a dedicated resource has been developed [4], which has embedded the possibility of analyzing real data from the ATLAS experiment. The HY.P.A.T.I.A tool which is used for this analysis is described in the next section.

\section{Description of the "HYPATIA" data analysis tool}

HY.P.A.T.I.A (Hybrid Pupil's Analysis Tool for Interactions in ATLAS), has been developed at the National and Kapodistrian University of Athens by two of the authors (C.K and S.V) and offers the user the ability to visualize and analyse events recorded by the ATLAS experiment.

Two different versions of the tool exist: the off-line version which is used within the large scale initiative called International Masterclasses [5] (full day duration) since 2008 by thousands of high-school students in all continents and the on-line one [6]. The on-line version is simpler to use, yet contains all the functionalities necessary for performing the various scenaria associated with a series of challenges in which students are involved. In 2016 the on-line version of HYPATIA was awarded the GOLC award [7] for the "best visualized experiment".

The on-line version has several advantages including its ability to be used on mobile devices (smartphones and tablets), ease of use (no installation or file downloads necessary) and event batch processing capability. HYPATIA represents the data graphically as event tracks on two separate views of the ATLAS detector and also includes all track details in the Track Momenta table (Figure 2).

The activities which the students are asked to perform during the implementation are to identify different kinds of leptons (electrons and muons) and then search for Z or Higgs boson leptonic decays by combining the products of their decay. This way the mass and width of the parent particle can be measured. This is a simplified but quite accurate view of actual researchers' work and aims to demonstrate current practices in high-end research as well as stimulate student interest towards particle physics. 


\section{Students' Evaluation}

The work of students is monitored step by step by a specially designed integrated assessment system which consists of three different layers.

- A figurative test, delivered before and after the activity, to measure whether the students' exposure to particle research has helped them recognize different patterns in the provided shapes.

- A set of assessment questions in each of the four phases of the scenario which uses as a reference the PISA 2012 Framework [8] developed for the assessment of problem solving competence (PSQ).

- A number of indicators embedded in the HYPATIA on-line analysis tool, which provide information about the ability of the students to understand and handle the complex analysis of short lived particle signatures in the state-of-the-art ATLAS detector.

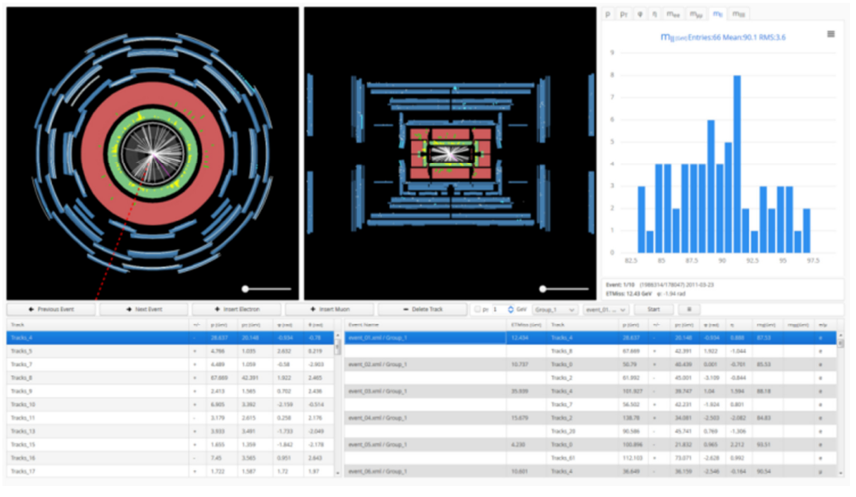

Figure 2. HYPATIA event analysis tool (online version)

The highest statistics sample is provided by the PSQ questions, namely the answers to the questions which are required at the end of each of the first four phases of the educational resource. The students are asked to answer two questions in each phase, eight in total. This way the authors are able to assess the students' performance by assessing their partial abilities in solving complex problems. Three different answers to the questions are proposed as an option and all of them are correct. However their level of complexity, completeness and accuracy varies and have thus been classified (following the PISA Framework for assessing the problem solving competence of students) as Low, Medium and High. The items have been standardized towards PISA items used in 2012 PISA study in order to allow for comparisons. This process allows teachers to not only assess the performance of the students' abilities but also to compare the level of the class with national or international standards. Additionally such an approach can be used in a series of challenges and lessons in order to enable the teachers to assess the progressive development of the classroom.

The proposed scenaria have been implemented in 42 different school environments (urban, sub-urban and rural schools) during a period of two school years 2015-2017 in the framework of the project work that is part of the Greek National Curriculum. The implementation involved both the Let's Accelerate Particles and the HYPATIA resource. About 570 students' groups (the students work in pairs at the school computer labs) took part. The results which are shown in Figure 3 clearly indicate that the students are scoring quite high demonstrating that the appropriate guidance was provided by the intervention and the effectiveness of the approach and the tools used. 


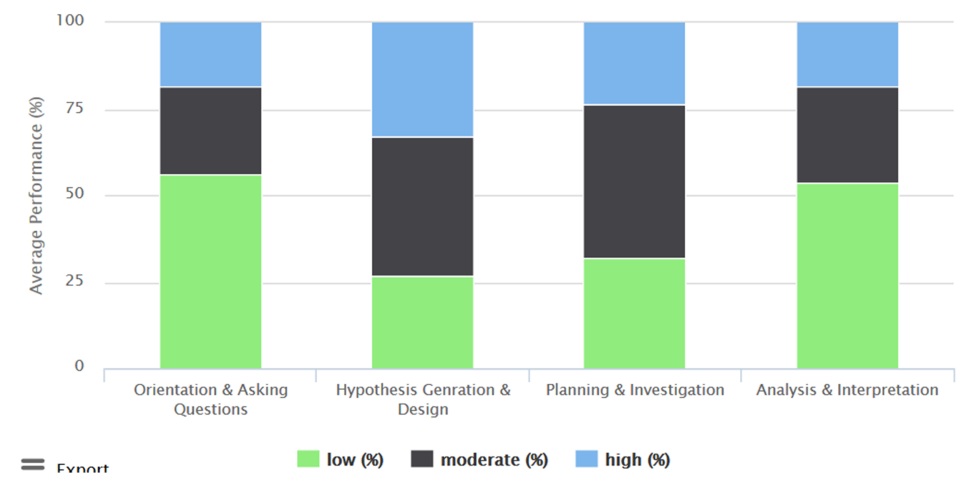

Figure 4. Students' performance in the PSQ questions of the four phases of the educational scenaria "Let's accelerate particles" and "HYPATIA demonstrator"

leptonic decays, half of them to $\mathrm{e}^{+} \mathrm{e}^{-}$pairs and half of them to $\mu^{+} \mu^{-}$pairs. The number of $\mathrm{Z}$ bosons found divided by the time available for the exercise -since sometimes the time available

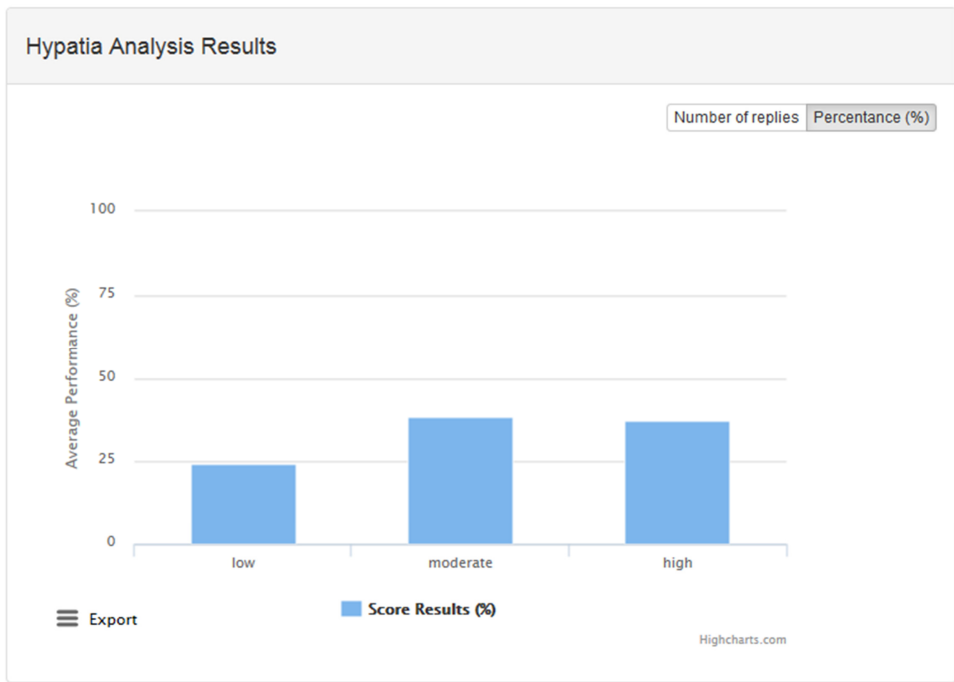

Figure 3. Students' evaluation results using the specially designed HYPATIA tool indicators

not real but are created by combining two $\mathrm{Z}$ boson events. Each indicator is recorder separately and their combination is shown on Figure 4.

\section{Resources for University students}

Since there have been very few resources targeted at university students, we have added a new functionality to the HYPATIA tool which simulates even more accurately the researchers' investigations for discoveries by processing thousands of events. During the duration of a lab class we encourage the university students to optimize the values of parameters which better select the signal over the background. To that end both simulated signal and background events, as well as the recently released real data of $1 \mathrm{fb}^{-1}$ from the ATLAS Open Data [9] are used. 


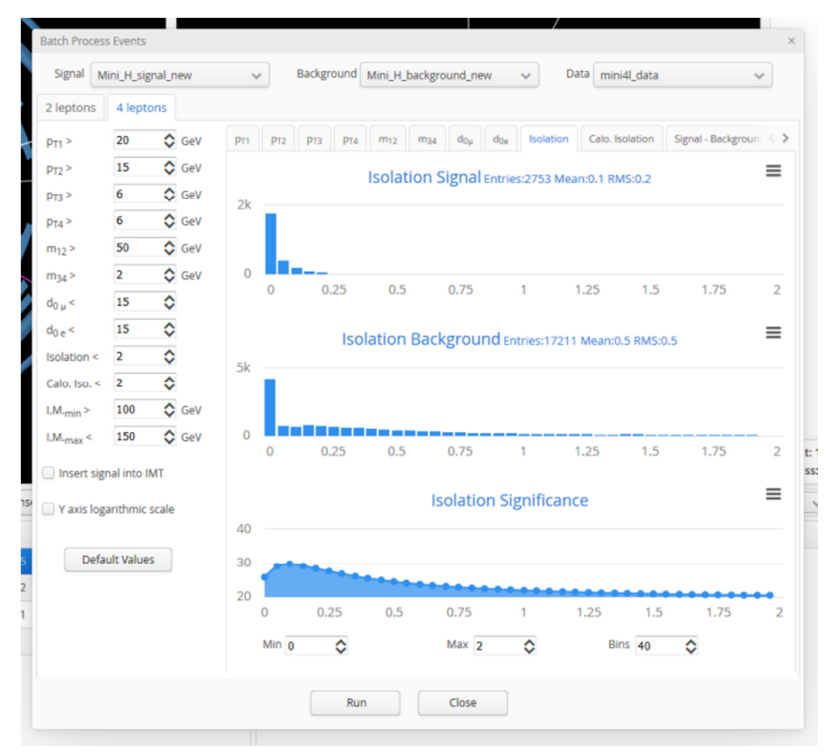

Figure 5. An example of the track isolation cut optimization using the HYPATIA on-line batch analysis tool
Figure 5 is an example of optimizing the track isolation cut by displaying the distribution of simulated Higgs signal events (top graph), simulated background events (middle graph) and the signal significance (bottom graph). The optimum value maximizes the significance. The student goes through several iterations in order to optimize all the cuts shown on the left side of Figure 5. This exercise has been successfully used in the undergraduate laboratory for third year students specializing in particle physics at

the University of Athens and also as an undergraduate laboratory exercise at the University of Birmingham with great success and very positive feedback from

the users. The relevant link is included in the ATLAS Open Data external resource page [10].

\section{Conclusions}

A variety of educational scenaria for involving students to HEP have been developed within the framework of the CREATIONS project and a number of interventions in the form of a mini masterclass have taken place in Greece in urban, suburban and rural schools. Different resources have been developed for different age groups of students including one for university students. The events have been very successful and students' feedback has been very positive.

The data analysis of the three different layers of assessment tools demonstrates that the interventions in the classrooms have followed the planned format (activities organisation, resources used, time devoted per inquiry phase). In addition the results provide clear indication that the students are demonstrating deep involvement and high problem solving skills. The students' scores out-perform the OECD average scores even after the application of quite demanding problem solving tasks.

\section{Acknowledgements}

The research presented in this article is funded by the European Union in the context of the CREATIONS project (Grant Agreement no. 665917) under the "Science with and for Society" section of the Horizon 2020 Work Programme. This document does not represent the opinion of the European Union, and the European Union is not responsible for any use that might be made of its content. 


\section{References}

[1] [Online] http://creations-project.eu/

[2] [Online] http://tools.inspiringscience.eu/delivery/view/index.html?id=02afe 7b3264f4848bf59c42a6b07e3cf\& $\mathrm{t}=\mathrm{p}$

[3] [Online] http://tools.inspiringscience.eu/delivery/view/index.html?id=c4e9fd501dce4f6290d35f8dcb9dcfld\& $\mathrm{t}=\mathrm{p}$

[4] [Online] http://tools.inspiringscience.eu/delivery/view/index.html?id=41 fbf7bc710a470aa531911f744973cf\& $\mathrm{t}=\mathrm{p}$

[5] [Online] http://www.physicsmasterclasses.org

[6] C. Kourkoumelis \& S. Vourakis (2014). Hypatia—an online tool for ATLAS event visualization. Physics Education, 49, 21-32

[7] Global Online Laboratory Consortium: http://online-engineering.org/GOLC_about.php

[8] OECD (2014) PISA 2012 Results: Creative Problem Solving: Students'Skills in Tackling Real-Life Problems (Volume V), PISA, OECD Publishing, p.31 doi:10.1787/9789264208070-en

[9] [Online] http://opendata.atlas.cern/

[10] [Online] http://opendata.atlas.cern/externals/ 